\title{
Injections of Artin groups
}

\author{
Robert W. Bell and Dan Margalit*
}

\begin{abstract}
We study those Artin groups which, modulo their centers, are finite index subgroups of the mapping class group of a sphere with at least 5 punctures. In particular, we show that any injective homomorphism between these groups is given by a homeomorphism of a punctured sphere together with a map to the integers. The technique, following Ivanov, is to prove that every superinjective map of the curve complex of a sphere with at least 5 punctures is induced by a homeomorphism. We also determine the automorphism group of the pure braid group on at least 4 strands.
\end{abstract}

Mathematics Subject Classification (2000). Primary 20F36; Secondary 57M07.

Keywords. Artin groups, mapping class groups, curve complex, co-Hopfian.

\section{Introduction}

We investigate injective homomorphisms between Artin groups which, modulo their centers, embed as finite index subgroups in the mapping class group of an $m$-times punctured sphere $S_{m}$, where $m \geq 5$.

The extended mapping class group of a surface $F$ is the group of isotopy classes of homeomorphisms of $F$ :

$$
\operatorname{Mod}(F)=\pi_{0}(\operatorname{Homeo}(F)) .
$$

Theorem 1. Let $m \geq 5$. If $G$ is a finite index subgroup of $\operatorname{Mod}\left(S_{m}\right)$ and $\rho: G \rightarrow$ $\operatorname{Mod}\left(S_{m}\right)$ is an injective homomorphism, then there is a unique $f \in \operatorname{Mod}\left(S_{m}\right)$ so that $\rho(g)=f g f^{-1}$ for all $g \in G$.

In particular, Theorem 1 applies to four infinite families of Artin groups modulo their centers: $A\left(A_{n}\right) / Z, A\left(B_{n}\right) / Z, A\left(\tilde{C}_{n-1}\right)$, and $A\left(\tilde{A}_{n-1}\right)$ where $n=m-2$ (see below for definitions). The group $A\left(B_{n}\right)$ is a subgroup of $A\left(A_{n}\right)$ and inherits the same center $Z$; the groups $A\left(\tilde{C}_{n-1}\right)$ and $A\left(\tilde{A}_{n-1}\right)$ have trivial center. Thus, Theorem 1 is a

* The first author is partially supported by a VIGRE postdoctoral position under NSF grant number 0091675 to the University of Utah. The second author is supported by an NSF postdoctoral fellowship. 
generalization of work of Charney-Crisp, who computed the automorphism groups of the aforementioned Artin groups using similar techniques [9].

Following Ivanov, we prove Theorem 1 by translating the problem into one about the curve complex $\mathcal{C}\left(S_{m}\right)$. This is the abstract simplicial flag complex with vertices corresponding to isotopy classes of essential curves in $S_{m}$ and edges corresponding to disjoint pairs of curves. To this end, we focus on particular elements of $G$ : powers of Dehn twists; each such element is associated to a unique isotopy class of curves in $S_{m}$ (see Section 2). We show that the injection $\rho$ must take powers of Dehn twists to powers of Dehn twists, thus giving an action $\rho_{\star}$ on the vertices of $\mathcal{C}\left(S_{m}\right)$. Since $\rho_{\star}$ is easily seen to be superinjective in the sense of Irmak (i.e. $\rho_{\star}$ preserves disjointness and nondisjointness), we will be able to derive Theorem 1 from the following theorem, proven in the appendix.

Theorem 2. Let $m \geq 5$. Every superinjective map of $\mathcal{C}\left(S_{m}\right)$ is induced by a unique element of $\operatorname{Mod}\left(S_{m}\right)$.

The proofs of both theorems are modeled on work of Ivanov, who showed that every isomorphism between finite index subgroups of $\operatorname{Mod}(F)$ is the restriction of an inner automorphism of $\operatorname{Mod}(F)$, when the genus of $F$ is at least 3 [24]. To do this, he applied his theorem that every automorphism of $\mathcal{C}(F)$ is induced by an element of $\operatorname{Mod}(F)$. His method has been used to prove similar theorems by various other authors ([26], [28], [18], [20], [19], [15], [31], [8]). In particular, Korkmaz proved that every automorphism of $\mathcal{C}\left(S_{m}\right)$ is induced by an element of $\operatorname{Mod}\left(S_{m}\right)$ [26], and Irmak showed that every superinjective map of $\mathcal{C}(F)$, for higher genus $F$, is induced by an element of $\operatorname{Mod}(F)$, thus obtaining the analog of Theorem 1 for surfaces of genus at least 2 ([18], [20], [19]).

After the completion of the work presented in this paper, the final cases of Irmak's theorem were completed by Behrstock-Margalit [3] and Shackleton [34]. See the appendix for a detailed historical remark.

Artin groups. Before we explain the applications of Theorem 1 to Artin groups, we recall the basic definitions. An Artin group is any group with a finite set of generators $\left\{s_{1}, \ldots, s_{n}\right\}$ and, for each $i \neq j$, a defining relation of the form

$$
s_{i} s_{j} \ldots=s_{j} s_{i} \ldots
$$

where $s_{i} s_{j} \ldots$ denotes an alternating string of $m_{i j}=m_{j i}$ letters. The value of $m_{i j}$ must lie in the set $\{2,3, \ldots, \infty\}$ with $m_{i j}=\infty$ signifying that there is no defining relation between $s_{i}$ and $s_{j}$.

It is convenient to define an Artin group by a Coxeter graph, which has a vertex for each generator $s_{i}$ and an edge labelled $m_{i j}$ connecting the vertices corresponding to $s_{i}$ and $s_{j}$ if $m_{i j}>2$. The label 3 is suppressed. The Coxeter graphs $A_{n}, B_{n}, \tilde{C}_{n-1}$, 
and $\tilde{A}_{n-1}$ for the Artin groups $A\left(A_{n}\right), A\left(B_{n}\right), A\left(\tilde{C}_{n-1}\right)$, and $A\left(\tilde{A}_{n-1}\right)$ are displayed in Figure 1.

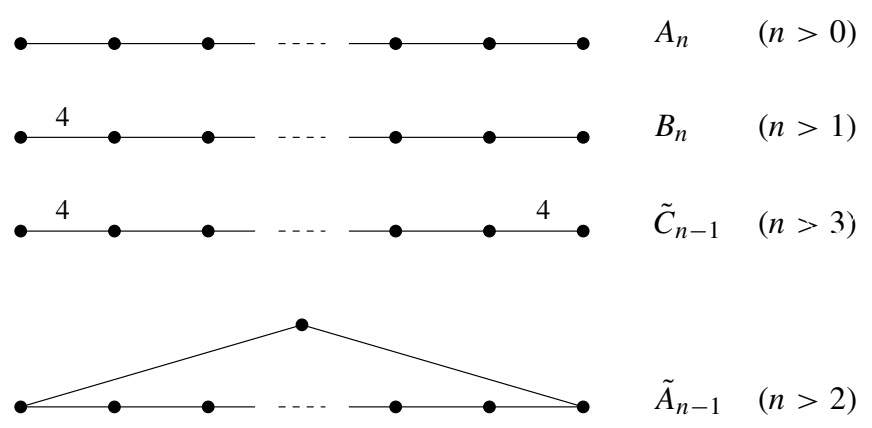

Figure 1. Coxeter graphs with $n$ vertices.

Artin groups and mapping class groups. The Artin group $A\left(A_{n}\right)$ is better known as the braid group on $n+1$ strands. If $D_{n+1}$ is the disk with $n+1$ punctures, and Homeo $\left(D_{n+1}, \partial D_{n+1}\right)$ is the space of homeomorphisms of $D_{n+1}$ which are the identity on the boundary, then $A\left(A_{n}\right)$ is isomorphic to

$$
\operatorname{Mod}\left(D_{n+1}, \partial D_{n+1}\right)=\pi_{0}\left(\operatorname{Homeo}\left(D_{n+1}, \partial D_{n+1}\right)\right)
$$

(see, e.g., [6]). Note that homeomorphisms which are the identity on the boundary are necessarily orientation preserving. The pure braid group $P\left(A_{n}\right)$ is the (finite index) subgroup of $A\left(A_{n}\right)$ consisting of elements which fix each puncture of $D_{n+1}$. The group $A\left(B_{n}\right)$ is isomorphic to a subgroup of $A\left(A_{n}\right)$ fixing one given puncture (see [1] or [9]).

The center $Z$ of $A\left(A_{n}\right)$ is generated by the Dehn twist about a curve isotopic to $\partial D_{n+1}$; we denote this element by $z$. Both $A\left(B_{n}\right)$ and $P\left(A_{n}\right)$ inherit the same center.

We can also identify $A\left(A_{n}\right) / Z, A\left(B_{n}\right) / Z$, and $A\left(\tilde{C}_{n-1}\right)$ with the subgroups of $\operatorname{Mod}\left(S_{n+2}\right)$ consisting of orientation preserving elements which fix one, two, and three particular punctures, respectively; further $P\left(A_{n}\right) / Z$ is isomorphic to the pure mapping class group $\operatorname{PMod}\left(S_{n+2}\right)$, which is the finite index subgroup of $\operatorname{Mod}\left(S_{n+2}\right)$ consisting of orientation preserving elements which fix every puncture. The group $A\left(\tilde{A}_{n-1}\right)$ is also isomorphic to a finite index subgroup of $\operatorname{Mod}\left(S_{n+2}\right)$. A complete description of these isomorphisms appears in the paper of Charney-Crisp [9]. The proofs are due to Allcock, Kent-Peifer, Charney-Peifer, Crisp, and Charney-Crisp ([1], [25], [10], [12], [9]).

Applications. We now give some consequences of Theorem 1. A group is co-Hopfian if each of its injective endomorphisms is an isomorphism. 
Corollary 3. For $n \geq 3$, all finite index subgroups of $\operatorname{Mod}\left(S_{n+2}\right)$ are co-Hopfian; in particular, the groups $A\left(A_{n}\right) / Z, A\left(B_{n}\right) / Z, A\left(\tilde{C}_{n-1}\right), A\left(\tilde{A}_{n-1}\right)$, and $P\left(A_{n}\right) / Z$ are co-Hopfian.

For each $0 \leq k \leq m=n+2$, let $G_{k}$ be a subgroup of $\operatorname{Mod}\left(S_{m}\right)$ consisting of orientation preserving elements which fix $k$ given punctures. Note that $G_{0}$ is the index 2 subgroup of $\operatorname{Mod}\left(S_{m}\right)$ consisting of orientation preserving elements, $G_{1} \cong A\left(A_{n}\right) / Z$, and $G_{m-1}=G_{m}=\operatorname{PMod}\left(S_{m}\right)$. Also, $G_{2} \cong A\left(B_{n}\right) / Z$ and $G_{3} \cong A\left(\tilde{C}_{n-1}\right)$.

Corollary 4. Suppose $n \geq 3$ and let $G$ and $H$ be any of the groups in Figure 2. Then there exists an injection $\rho: G \rightarrow H$ if and only if there is directed path from $G$ to $H$ in Figure 2.

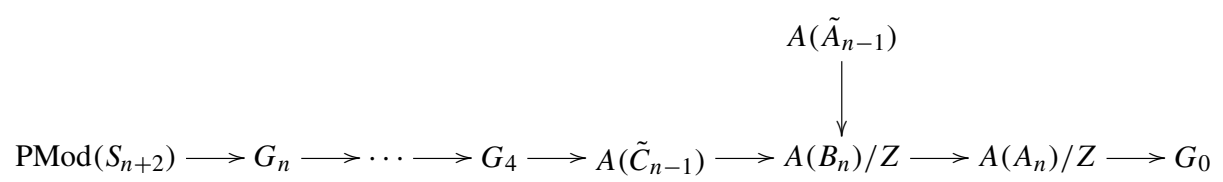

Figure 2. The diagram for Corollary 4.

Theorem 1 tells us that $\rho$ preserves the index of $G$. We have $\left[\operatorname{Mod}\left(S_{m}\right): G_{k}\right]=$ $2 m ! /(m-k)$ !, and the index of $A\left(\tilde{A}_{n-1}\right)$ is the same as $A\left(\tilde{C}_{n-1}\right) \cong G_{3}$ (see [9]). Since $A\left(\tilde{A}_{n-1}\right) \supsetneqq A\left(\tilde{C}_{n-1}\right)$ (see [9]), there are no arrows from right to left. To complete the proof of the corollary, it suffices to note that $P\left(A_{n}\right) / Z$ is not a subgroup of $A\left(\tilde{A}_{n-1}\right)$ (see [9]) and that $P\left(A_{n}\right) / Z$, contained in each $G_{k}$, is normal in $\operatorname{Mod}\left(S_{m}\right)$.

One might also ask whether or not any injection from Corollary 4 is unique up to automorphisms of $H$. The answer is no. For instance, since $G_{m}$ is normal in $G_{0}$, we may conjugate $G_{m}$ by any element of $\operatorname{Mod}\left(S_{m}\right)$ to get an injective homomorphism $G_{m} \rightarrow G_{k}$ for any $k$. However, if $k>0$, then $f$ might not fix the $k$ punctures fixed by $G_{k}$, and so there is no automorphism of $G_{k}$ which achieves the injection.

We also characterize injections between the groups $A\left(A_{n}\right), A\left(B_{n}\right)$, and $P\left(A_{n}\right)$ (with their centers). There are inclusions: $P\left(A_{n}\right) \rightarrow A\left(B_{n}\right) \rightarrow A\left(A_{n}\right)$ (see Section 5); all other injections between these groups are described by the following corollary to Theorem 1 . We denote the center of a group $G$ by $Z(G)$.

Theorem 5. Suppose $n \geq 3$ and let $G$ be a finite index subgroup of $A\left(A_{n}\right)$. If $\rho: G \rightarrow A\left(A_{n}\right)$ is an injective homomorphism, then there is an induced injection $G / Z(G) \rightarrow A\left(A_{n}\right) / Z$. Moreover, there is a unique $f \in \operatorname{Mod}\left(S_{n+2}\right)$ so that, after identifying $A\left(A_{n}\right) / Z$ with the group $G_{1}$, we have

$$
\rho(g) Z=f(g Z) f^{-1}
$$

for all $g \in G$. 
In Section 5, we explain how this theorem may be applied to give an explicit list of all injections of $A\left(A_{n}\right), A\left(B_{n}\right)$, and $P\left(A_{n}\right)$ into $A\left(A_{n}\right)$. The case of $A\left(A_{n}\right)$ was already handled in a previous paper of the authors [4].

Combining Theorem 5 with Corollary 4, we immediately obtain an analogue of Corollary 4 for $A\left(A_{n}\right)$. Precisely, if $L_{k}$ is a subgroup of $A\left(A_{n}\right)$ corresponding to elements which fix $k$ particular punctures, then there is an injective homomorphism $L_{j} \rightarrow L_{k}$ if and only if $j>k$. In particular, there is an injective homomorphism between two of the groups $A\left(A_{n}\right), A\left(B_{n}\right)$, and $P\left(A_{n}\right)$ if and only if there is an obvious one.

We further prove the following theorem. For the statement, note that each $L_{k}$ inherits the same center $Z$ as the entire group $A\left(A_{n}\right)$.

Theorem 6. The natural map $\operatorname{Aut}\left(L_{k}\right) \rightarrow \operatorname{Aut}\left(L_{k} / Z\right)$ is surjective.

We give a more complete description of $\operatorname{Aut}\left(L_{k}\right)$ in two cases. The first is a theorem of Charney-Crisp [9].

Theorem 7. For $n \geq 3$, we have

$$
\operatorname{Aut}\left(A\left(B_{n}\right)\right) \cong\left(\mathbb{Z}_{2} \times \mathbb{Z}_{2}\right) \ltimes\left(G_{2} \times \mathbb{Z}\right)
$$

where $G_{2}$ is the group defined above.

Whereas the Charney-Crisp proof of Theorem 7 relies on a semidirect product decomposition of $A\left(B_{n}\right)$ due to Kent-Peifer, we work directly from the isomorphism $A\left(B_{n}\right) \cong L_{1}$.

Finally, we compute the automorphism group of the pure braid group. The proof uses our understanding of injections $P\left(A_{n}\right) \rightarrow A\left(A_{n}\right)$, a theorem of Korkmaz, and a direct product decomposition of $P\left(A_{n}\right)$ pointed out to us by Luis Paris.

Theorem 8. Suppose $n \geq 3$ and let $N=\left(\begin{array}{c}n+1 \\ 2\end{array}\right)$. We have

$$
\operatorname{Aut}\left(P\left(A_{n}\right)\right) \cong \operatorname{Mod}\left(S_{n+2}\right) \ltimes\left(\mathbb{Z}_{2} \ltimes \mathbb{Z}^{N-1}\right) .
$$

We note that $P\left(A_{2}\right)$ is isomorphic to the free group on two letters and $P\left(A_{1}\right) \cong \mathbb{Z}$.

Acknowledgements. The authors would like to thank Benson Farb for bringing the work of Charney-Crisp to our attention and for his continued encouragement on this project. Joan Birman, Chris Leininger, and Luis Paris were very generous with their time and energy; for this we are thankful. We are also grateful to Mladen Bestvina, Tara Brendle, Ruth Charney, Feng Luo, Andy Putman, Michah Sageev, Saul Schleimer, Steven Spallone, and Kevin Wortman for helpful conversations. Finally, we thank the referees for comments which improved the paper. 


\section{Background}

Curves. By a curve in a surface $F$, we mean the isotopy class of a simple closed curve in $F$ which is not isotopic to a point, a puncture, or a boundary component of $F$. We will often not make the distinction between a representative curve and its isotopy class.

We denote by $i(a, b)$ the geometric intersection number between two curves $a$ and $b$.

A maximal collection of pairwise disjoint curves in $S_{m}$ is called a pants decomposition. Any pants decomposition of $S_{m}$ or $D_{m-1}$ has $m-3$ curves.

The interior of a curve in $D_{n+1}$ is the component of its complement which does not contain $\partial D_{n+1}$. A curve in $D_{n+1}$ with $k$ punctures in its interior is called a $k$-curve.

Curve complex. The curve complex $\mathcal{C}(F)$ for a surface $F$, defined by Harvey, is the abstract simplicial flag complex with a vertex for each curve in $F$ and edges corresponding to geometric intersection zero [17].

A map $\phi: \mathcal{C}(F) \rightarrow \mathcal{C}(F)$ is called superinjective if for any two vertices $v$ and $w$ of $\mathcal{C}(F)$, thought of as curves in $F$, we have that $i(v, w)=0$ if and only if $i(\phi(v), \phi(w))=0$. Superinjective maps of $\mathcal{C}\left(S_{m}\right)$ are injective for $m \geq 5$ since, given two distinct curves, there is a curve which is disjoint from one but not the other.

Twists. A Dehn twist about a curve $a$, denoted $T_{a}$, is the element of the mapping class group which has support on an annular neighborhood of $a$, and is described on that annulus by Figure 3.

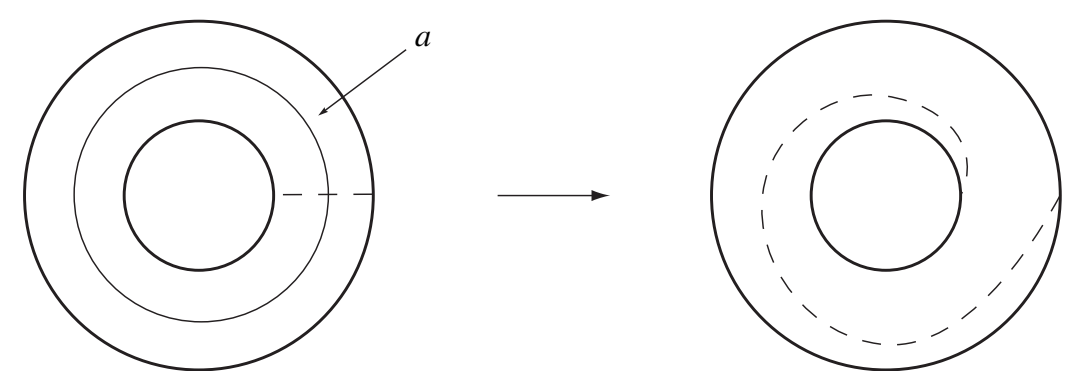

Figure 3. Dehn twist about a curve $a$.

If $a$ is a 2-curve, we define the half twist about $a$, denoted $H_{a}$, to be the element of the mapping class group which has support the interior of $a$, and is described inside this twice-punctured disk by Figure 4.

For each $f \in \operatorname{Mod}\left(S_{m}\right)$, let $\varepsilon(f)=1$ if $f$ preserves orientation and $\varepsilon(f)=-1$ if not. We will use the following connection between the topology and algebra of Dehn 


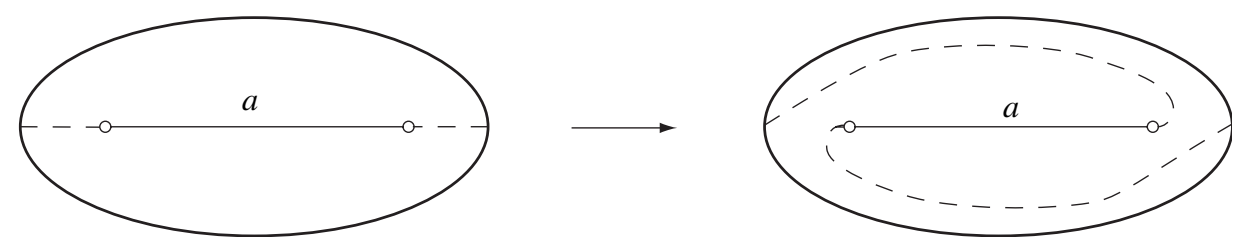

Figure 4. Half twist about a curve $a$.

twists in $\operatorname{Mod}\left(S_{m}\right)$. In the statement (and throughout the paper), we use functional notation; that is, elements are applied right to left.

Fact 9. Suppose $f \in \operatorname{Mod}\left(S_{m}\right)$. Then $f T_{a} f^{-1}=T_{f(a)}^{\varepsilon(f)}$. In particular, $\left[f, T_{a}\right]=1$ implies $f(a)=a$, and powers of Dehn twists commute if and only if the curves have geometric intersection zero.

For a group $\Gamma$, we define its rank, $\operatorname{rk} \Gamma$, to be the maximal rank of a free abelian subgroup of $\Gamma$. It follows from work of Birman-Lubotzky-McCarthy that for any surface $F$, rk $\operatorname{Mod}(F)$ is realized by any subgroup generated by powers of Dehn twists about curves forming a pants decomposition for $F$ [7]; thus, $\operatorname{rk} \operatorname{Mod}\left(S_{m}\right)=m-3$. The following theorem of Ivanov gives another connection between the algebra and topology of $\operatorname{Mod}\left(S_{m}\right)$ [24]. We restrict our attention here to the genus 0 case, which has a particularly simple statement. Throughout, $C_{H}(g)$ denotes the centralizer of $g$ in the group $H$.

Theorem 10. Let $m \geq 5$ and let $P$ be a finite index subgroup of $\operatorname{PMod}\left(S_{m}\right)$. An element $g$ of $P$ is a power of Dehn twist if and only if $Z\left(C_{P}(g)\right) \cong \mathbb{Z}$ and $\operatorname{rk} C_{P}(g)=$ $m-3$.

We now state a group theoretical lemma, due to Ivanov-McCarthy [21], which will be used in Proposition 12.

Lemma 11. Let $\rho: \Gamma \rightarrow \Gamma^{\prime}$ be any injective homomorphism of groups, where $\mathrm{rk} \Gamma^{\prime}=$ $\mathrm{rk} \Gamma<\infty$. If $G<\Gamma$ is a free abelian subgroup of maximal rank, and $g \in G$, then

$$
\operatorname{rk} Z\left(C_{\Gamma^{\prime}}(\rho(g))\right) \leq \operatorname{rk} Z\left(C_{\Gamma}(g)\right) .
$$

Note that Lemma 11 applies whenever $g$ is a power of a Dehn twist and both $\Gamma$ and $\Gamma^{\prime}$ are finite index subgroups of $\operatorname{PMod}\left(S_{m}\right)$. 


\section{Subgroups of $\operatorname{Mod}\left(S_{m}\right)$}

Let $\rho: G \rightarrow \operatorname{Mod}\left(S_{m}\right)$ be an injective homomorphism, where $G$ is a finite index subgroup of $\operatorname{Mod}\left(S_{m}\right)$ with $m \geq 5$. Set $Q=\operatorname{PMod}\left(S_{m}\right)$ and $P=Q \cap \rho^{-1}(Q)$. Note that since $P$ is a finite index subgroup of $\operatorname{Mod}\left(S_{m}\right)$, every Dehn twist has a nontrivial power in $P$.

Proposition 12. Let a be a curve in $S_{m}$, and choose any nonzero $k$ so that $T_{a}^{k} \in P$. There is a unique curve $a^{\prime}$ (independent of $k$ ) and an integer $k^{\prime}$ so that $\rho\left(T_{a}^{k}\right)=T_{a^{\prime}}^{k^{\prime}}$.

Proof. By Theorem 10, $Z\left(C_{P}(g)\right) \cong \mathbb{Z}$. Lemma 11 and the fact that $\rho$ is injective imply that $Z\left(C_{Q}(\rho(g))\right) \cong \mathbb{Z}$. Since $\mathrm{rk} \rho\left(C_{P}(g)\right)=\operatorname{rk} \operatorname{Mod}\left(S_{m}\right)$, Theorem 10 says that $\rho(g)$ must be a power of a Dehn twist about some curve $a^{\prime}$. The uniqueness of $a^{\prime}$ follows from the fact that $\left\langle T_{a}\right\rangle \cap P$ is cyclic.

By Proposition 12, $\rho$ induces a well-defined action $\rho_{\star}$ on curves given by

$$
\rho\left(T_{a}^{k}\right)=T_{\rho_{\star}(a)}^{k^{\prime}} .
$$

Applying Fact 9, we have:

Proposition 13. The map $\rho_{\star}$ is a superinjective map of $\mathcal{C}\left(S_{m}\right)$.

We are now ready to complete the proof of Theorem 1, assuming Theorem 2.

Proof. By Propositions 12 and 13, the injection $\rho$ gives rise to a superinjective map $\rho_{\star}$ of $\mathcal{C}(S)$, which by Theorem 2 is induced by a unique $f \in \operatorname{Mod}\left(S_{m}\right)$; that is to say, $\rho_{\star}(c)=f(c)$ for every curve $c$. Since $f$ is unique, we can check that $\rho(g)=f g f^{-1}$ by checking that $f g(c)=\rho(g) f(c)$ for any curve $c$. We choose $k$ so that $T_{g(c)}^{k}, T_{c}^{k} \in P$, and let $k^{\prime}$ and $k^{\prime \prime}$ be the integers given by the application of Proposition 12 to $T_{g(c)}^{k}$ and $T_{c}^{k}$.

$$
\begin{aligned}
T_{f g(c)}^{k^{\prime}} & =\rho\left(T_{g(c)}^{k}\right)=\rho\left(g T_{c}^{ \pm k} g^{-1}\right)=\rho(g) \rho\left(T_{c}^{ \pm k}\right) \rho(g)^{-1} \\
& =\rho(g) T_{f(c)}^{k^{\prime \prime}} \rho(g)^{-1}=T_{\rho(g) f(c)}^{ \pm k^{\prime \prime}} .
\end{aligned}
$$

Thus $T_{f g(c)}^{k^{\prime}}=T_{\rho(g) f(c)}^{ \pm k^{\prime \prime}}$, which implies that $f g(c)=\rho(g) f(c)$.

\section{Subgroups of $A\left(A_{n}\right)$}

Let $G<A\left(A_{n}\right)$ be a finite index subgroup and $\rho: G \rightarrow A\left(A_{n}\right)$ an injective homomorphism. To prove Theorem 5, we need to show that $\rho$ induces an injective homomorphism $G / Z(G) \rightarrow A\left(A_{n}\right) / Z$ and apply Theorem 1 . 
As with Theorem 1, we shall require the existence of a superinjective map $\rho_{\star}$ of $\mathcal{C}\left(D_{n+1}\right)$ which is induced by $\rho$ in the sense that for any curve $a$ we have

$$
\rho\left(T_{a}^{k}\right)=T_{\rho_{\star}(a)}^{k^{\prime}} z^{k^{\prime \prime}}
$$

for some integers $k, k^{\prime}$, and $k^{\prime \prime}$ ( $k$ and $k^{\prime}$ nonzero); as usual $z$ is the generator of the center of $A\left(A_{n}\right)$. The argument is exactly the same as in Proposition 12, with Theorem 10 replaced by the following corollary of Theorem 10 .

Corollary 14. Let $P$ be a finite index subgroup of $P\left(A_{n}\right)$. An element $g$ of $P$ is the product of a central element and a nontrivial power of a noncentral Dehn twist if and only if $Z\left(C_{P}(g)\right) \cong \mathbb{Z}^{2}$ and $\operatorname{rk} C_{P}(g)=n$.

We now prove the theorem.

Proof of Theorem 5. Let $G$ be a finite index subgroup of $A\left(A_{n}\right)$ and $\rho: G \rightarrow A\left(A_{n}\right)$ an injective homomorphism. We know that $G$ has nontrivial center $Z(G)$ since it is finite index in $A\left(A_{n}\right)$. Further we have $Z(G)=Z \cap G \cong \mathbb{Z}$. Indeed, if $\zeta$ is an element of $Z(G)$, then $\zeta$ must fix every curve in $D_{n+1}$ by Fact 9 and the fact that $G$ is finite index; hence $\zeta$ is a power of $z$.

Let $\zeta$ denote a generator of $Z(G)$. We now show that $\rho(Z(G))<Z$ by showing $\rho(\zeta) \in Z$. Since $\mathrm{rk} G=\operatorname{rk} A\left(A_{n}\right)$, we have that $\rho\left(\zeta^{k}\right) \in Z$ for some nonzero $k$.

Choose a pants decomposition $\mathcal{P}$ of $D_{n+1}$. As in Section 3, we know that $\rho_{\star}(\mathcal{P})$ is also a pants decomposition. Further, because $\zeta$ is central and $\rho$ is injective, it follows that $\rho(\zeta)$ fixes each element of $\rho_{\star}(\mathcal{P})$. Since an orientation preserving element of $\operatorname{Mod}\left(S_{3}\right)$ is determined by its action on the punctures, it follows that $\rho(\zeta)$ lies in the free abelian subgroup generated by half twists and Dehn twists in the curves of $\rho_{\star}(\mathcal{P})$. Since $\rho(\zeta)^{k} \in Z$, it now follows that $\rho(\zeta) \in Z$.

Moreover, we have that $\rho^{-1}(Z)<Z(G)$, by the injectivity of $\rho$. Thus, $\rho$ induces a well-defined injection $G / Z(G) \rightarrow A\left(A_{n}\right) / Z$. Since $G / Z(G)$ is finite index in $A\left(A_{n}\right) / Z$, we may apply Theorem 1 . Thus, fixing an identification $A\left(A_{n}\right) / Z<$ $\operatorname{Mod}\left(S_{n+2}\right)$, there is a unique $f \in \operatorname{Mod}\left(S_{n+2}\right)$ so that

$$
\rho(g) Z=f(g Z) f^{-1}
$$

for all $g \in G$. This proves the theorem.

We now take a moment to interpret Theorem 5 in a way that will be useful to us in the next section. The element $f \in \operatorname{Mod}\left(S_{n+2}\right)$ from the theorem does not necessarily correspond to an element of $\operatorname{Mod}\left(D_{n+1}\right)$, for it may switch the puncture of $S_{n+2}$ corresponding to the boundary of $D_{n+1}$ with another puncture. However, even in this case, since $\mathcal{C}\left(D_{n+1}\right) \cong \mathcal{C}\left(S_{n+2}\right)$, the element $f$ induces an automorphism $f_{\star}$ 
of $\mathcal{C}\left(D_{n+1}\right)$. Since $f$ is an element of $\operatorname{Mod}\left(S_{n+2}\right)$ as opposed to $\operatorname{Mod}\left(D_{n+1}\right)$, the map $f_{\star}$ may take a $k$-curve to an $(n-k+2)$-curve.

Now, let $g$ be a power of a noncentral Dehn twist or half twist in $G$; for concreteness, $g=T_{a}^{k}$. The coset $g Z$ is a power of a Dehn twist, also denoted $T_{a}^{k}$, thought of as an element of $\operatorname{Mod}\left(S_{n+2}\right)$. The conjugate $f(g Z) f^{-1}$ is equal to $T_{f_{\star}(a)}^{\varepsilon(f) k}$. It follows from Theorem 5 that $\rho(g)$ is a product of $T_{f_{\star}(a)}^{\varepsilon(f) k}$ with a central element. We again emphasize that $f$ is an element of the mapping class group of $S_{n+2}$, and not $D_{n+1}$, and so $f_{\star}$ can take a curve to one which is not topologically equivalent in $D_{n+1}$. In fact, we will see examples of this "nongeometric" phenomenon in the next section, where we classify injective homomorphisms of $A\left(A_{n}\right), A\left(B_{n}\right)$, and $P\left(A_{n}\right)$ into $A\left(A_{n}\right)$. As each of these groups is generated by half twists and Dehn twists, we will be able to understand these injections via the following corollary to Theorem 5, which summarizes the above discussion.

Corollary 15. Let $G$ be a finite index subgroup of $A\left(A_{n}\right)$ and $\rho: G \rightarrow A\left(A_{n}\right)$ an injective homomorphism. There is an $f \in \operatorname{Mod}\left(S_{n+2}\right)$ so that for any power of a Dehn twist $T_{a}^{k} \in G$, we have

$$
\rho\left(T_{a}^{k}\right)=T_{f_{\star}(a)}^{\varepsilon(f) k} z^{t}
$$

for some integer $t=t\left(T_{a}^{k}\right)$.

The analogous statement for half twists also holds. We remark that the reason we focus on Dehn twists and half twists here is that in $A\left(A_{n}\right)$ there is a natural representative of a Dehn twist coset of $A\left(A_{n}\right) / Z$, and so, combined with the action of $f_{\star}$ on curves, there is a relatively simple form for the image under $\rho$ of a power of a twist.

Moving punctures criterion. Another fact which will be useful in the next section is that $f$ must send moving punctures to moving punctures; that is, the set of punctures of $S_{n+2}$ which are not fixed by every element of $G / Z(G)$ must be sent by $f$ to into the $n+1$ punctures which are not fixed by $A\left(A_{n}\right) / Z$ (recall that only one puncture is fixed by $A\left(A_{n}\right) / Z$ ). This is because conjugation by $f$ sends fixed punctures to fixed punctures and moving punctures to moving punctures. Below, we call this the moving punctures criterion. We remark that this criterion can be used to derive Corollary 4 from Theorem 1.

\section{Catalogue of injections}

We now use Theorem 5 to list all injections of the groups $P\left(A_{n}\right), A\left(B_{n}\right)$, and $A\left(A_{n}\right)$ into $A\left(A_{n}\right)$. As usual, we denote the generator of $Z$ by $z$.

Instead of applying Theorem 5 directly, we will instead use Corollary 15 and the moving punctures criterion. We use the notation of Corollary 15: given an 
element $f$ of $\operatorname{Mod}\left(S_{n+2}\right)$, the symbol $f_{\star}$ denotes the induced automorphism of $\mathcal{C}\left(S_{n+2}\right)$; the identification $\mathcal{C}\left(D_{n+1}\right) \cong \mathcal{C}\left(S_{n+2}\right)$ comes from the identification $A\left(A_{n}\right) / Z<\operatorname{Mod}\left(S_{n+2}\right)$.

5.1. Injections of $\boldsymbol{A}\left(\boldsymbol{A}_{\boldsymbol{n}}\right)$. The Artin group $A\left(A_{n}\right)$ is defined via the presentation given by Figure 1 . We denote the generators by $\sigma_{1}, \ldots, \sigma_{n}$. Under the identification with $\operatorname{Mod}\left(D_{n+1}, \partial D_{n+1}\right)$, each generator $\sigma_{i}$ corresponds to a half twist $H_{a_{i}}$ about a curve $a_{i}$ in $D_{n+1}$ (see [6]).

Let $\rho: A\left(A_{n}\right) \rightarrow A\left(A_{n}\right)$ be an injective homomorphism. Applying the moving punctures criterion, we see that the element $f \in \operatorname{Mod}\left(S_{n+2}\right)$ given by Theorem 5 must send the puncture of $S_{n+2}$ fixed by $A\left(A_{n}\right) / Z$ to itself. Therefore, we may think of $f$ as an element of $\operatorname{Mod}\left(D_{n+1}\right)$. Corollary 15 then implies that $\rho$ is described on generators by the formula

$$
\rho\left(H_{a_{i}}\right)=H_{f_{\star}\left(a_{i}\right)}^{\varepsilon(f)} z^{t_{i}}
$$

where each $t_{i}$ is an integer. Since the $\sigma_{i}$ are all conjugate in $A\left(A_{n}\right)$, we have that $t_{i}$ is the same for all $i$. Conversely, any choices of $f \in \operatorname{Mod}\left(D_{n+1}\right)$ and $t=t_{1}$ determine an injective homomorphism. Indeed, Theorem 5 tells us that

$$
\rho(g) Z=f(g Z) f^{-1}
$$

and so the kernel of $\rho$ is contained in $Z$. However, since $z=\left(\sigma_{1} \ldots \sigma_{n}\right)^{n+1}$, we have

$$
\rho(z)=z \cdot z^{t(n(n+1))}=z^{1+t(n(n+1))} .
$$

As $n \geq 3$, we see $t(n(n+1))$ cannot be -1 , so $\rho(z)$ is not trivial, and the kernel of $\rho$ is trivial. Thus, we have an injection for any $t$; moreover, the map is not surjective when $t \neq 0$ : the preimage of $Z$ is contained in $Z$, but $z \mapsto z^{1+t(n(n+1))}$, so nothing maps to $z$.

It follows that $\operatorname{Aut}\left(A\left(A_{n}\right)\right) \cong \operatorname{Mod}\left(D_{n+1}\right)$. This was first proven by DyerGrossman [14]. Ivanov was the first to compute $\operatorname{Aut}\left(A\left(A_{n}\right)\right)$ from the perspective of mapping class groups [22].

5.2. Injections of $\boldsymbol{A}\left(\boldsymbol{B}_{\boldsymbol{n}}\right)$. Again, this group has a presentation given by Figure 1 . We denote the generators for $A\left(B_{n}\right)$, from left to right, by $s_{1}, \ldots, s_{n}$. The usual inclusion $A\left(B_{n}\right) \rightarrow A\left(A_{n}\right)$ is given by $s_{1} \mapsto \sigma_{1}^{2}$ and $s_{i} \mapsto \sigma_{i}$ for $i>1$.

Let $\rho: A\left(B_{n}\right) \rightarrow A\left(A_{n}\right)$ be an injective homomorphism. There are two punctures fixed by $A\left(B_{n}\right) / Z<\operatorname{Mod}\left(S_{n+2}\right)$. By the moving punctures criterion, the element $f$ given by Theorem 5 must send one of these two punctures to the puncture fixed by $A\left(A_{n}\right) / Z$. Identifying $\sigma_{i}$ with $H_{a_{i}}$ as above, this means that $f_{\star}$ takes $a_{i}$ to a 2-curve when $i>1$, and $f_{\star}\left(a_{1}\right)$ is either a 2-curve or an $n$-curve. We will see in Section 6 that $\operatorname{Aut}\left(A\left(B_{n}\right)\right) \rightarrow \operatorname{Aut}\left(A\left(B_{n}\right) / Z\right)$ is surjective, so we are forced to consider these nongeometric maps $f_{\star}$. 
As above, the homomorphism $\rho$ is given on generators by

$$
\rho\left(T_{a_{1}}\right)=T_{f_{\star}\left(a_{1}\right)}^{\varepsilon(f)} z^{t_{1}} \quad \text { and } \quad \rho\left(H_{a_{i}}\right)=H_{f_{\star}\left(a_{i}\right)}^{\varepsilon(f)} z^{t_{i}} \quad \text { for } i>1 .
$$

Since the $s_{i}$ are conjugate for $i>1$, we have that $t_{i}$ is the same for these $i$; set $u=t_{1}$ and $v=t_{2}$. Conversely, we have a well-defined homomorphism for any $u$ and $v$. If $g \mapsto 1$, we again have that $g \in Z$. Since $z=\left(s_{1} \ldots s_{n}\right)^{n}$, we have $z \mapsto z^{1+n u+n(n-1) v}$. But there are no $u$ and $v$ which make the latter trivial (as $n$ and $n(n-1)$ are not relatively prime), so every choice of $u$ and $v$ leads to an injection.

5.3. Injections of $\boldsymbol{P}\left(\boldsymbol{A}_{\boldsymbol{n}}\right)$. We identify $P\left(A_{n}\right)$ with the elements of $A\left(A_{n}\right)$ which fix each puncture of $D_{n+1}$. There is a standard generating set for $P\left(A_{n}\right)$, due to Artin, consisting of one Dehn twist $T_{a_{i, j}}$ for each pair of punctures of $D_{n+1}$ (see [29, pp. 173-174]). If the punctures of $D_{n+1}$ lie in a horizontal line, then each $a_{i, j}$ can be realized as the boundary of a regular neighborhood of an arc which lies below this horizontal and connects the $i^{\text {th }}$ and $j^{\text {th }}$ punctures; note that $a_{i}=a_{i, i+1}$.

Let $\rho: P\left(A_{n}\right) \rightarrow A\left(A_{n}\right)$ be an injective homomorphism. As in the previous cases, we apply Corollary 15 and deduce that $\rho$ is described on generators by

$$
\rho\left(T_{a_{i, j}}\right)=T_{f_{\star}\left(a_{i, j}\right)}^{\varepsilon(f)} z^{t_{i, j}}
$$

for some $f \in \operatorname{Mod}\left(S_{n+2}\right)$. In the case of $P\left(A_{n}\right) / Z$, there are no moving punctures in $S_{n+2}$, and so the moving punctures criterion gives no restriction for the action of $f$ on the punctures of $S_{n+2}$. We will see in Section 6 that in fact every $f \in \operatorname{Mod}\left(S_{n+2}\right)$ gives rise to an automorphism of $P\left(A_{n}\right)$, and so the $f$ associated to $\rho$ is arbitrary.

Conversely, since all of Artin's defining relations of $P\left(A_{n}\right)$ are commutation relations (see [2]), it follows that even if the $t_{i, j}$ are all different, $\rho$ is a well-defined homomorphism. Again, the kernel of $\rho$ must be contained in $Z$. In the generators of $P\left(A_{n}\right), z$ can be written as

$$
\left(T_{a_{n, n+1}}\right)\left(T_{a_{n-1, n+1}} T_{a_{n-1, n}}\right) \ldots\left(T_{a_{1, n+1}} \ldots T_{a_{1,3}} T_{a_{1,2}}\right)
$$

(recall we use functional notation) and so we see that

$$
z \mapsto z^{1+\sum t_{i, j}} .
$$

Hence, there is an affine hyperplane in $\mathbb{Z}^{N}$, where $N=\left(\begin{array}{c}n+1 \\ 2\end{array}\right)$, corresponding to noninjective homomorphisms of $P\left(A_{n}\right)$ into $A\left(A_{n}\right)$.

Remark. The abstract commensurator $\operatorname{Comm}(G)$ of a group $G$ is the collection of isomorphisms of finite index subgroups of $G$, where two such isomorphisms are equivalent if they agree on some common finite index subgroup. For a given group $A\left(A_{n}\right)$, $A\left(B_{n}\right)$, or $P\left(A_{n}\right)$, different choices of the function $t$ give rise to distinct elements of $\operatorname{Comm}\left(A\left(A_{n}\right)\right)$. After the first version of this paper was written, Leininger-Margalit proved that $\operatorname{Comm}\left(A\left(A_{n}\right)\right) \cong \operatorname{Mod}\left(S_{n+2}\right) \ltimes\left(\mathbb{Q}^{\times} \ltimes \mathbb{Q}^{\infty}\right)$ [27]. 


\section{Automorphisms}

In this section we prove Theorem 6 by constructing a lift $\xi_{k}$ of the natural map $\operatorname{Aut}\left(L_{k}\right) \rightarrow \operatorname{Aut}\left(L_{k} / Z\right)$. As in the introduction, $L_{k}$ is the subgroup of $A\left(A_{n}\right)$ consisting of elements which fix $k$ particular punctures. In the cases of $L_{1} \cong A\left(B_{n}\right)$ and $L_{n+1} \cong P\left(A_{n}\right)$, we will compute the automorphism groups explicitly (Theorems 7 and 8).

Before we begin in earnest, we note that any $L_{k}$ can be generated by Artin's generators for $P\left(A_{n}\right)$ plus a collection of half twists which are lifts of the elements of the symmetric group on $n+1$ letters which are in the image of $L_{k}$.

Charney-Crisp define a transvection of a group $G$ with infinite cyclic center $Z=\langle z\rangle$ to be a homomorphism $G \rightarrow G$ of the form $x \mapsto x z^{t(x)}$, where $t: G \rightarrow \mathbb{Z}$ is a homomorphism. They observe that such a map is an automorphism if and only if its restriction to $Z$ is surjective; this holds if and only if $t(z)= \pm 1$, i.e., $z \mapsto z^{ \pm 1}$. We denote by $\operatorname{Tv}(G)$ the transvection subgroup of $\operatorname{Aut}(G)$.

We consider the following sequence:

$$
1 \rightarrow \operatorname{Tv}\left(L_{k}\right) \rightarrow \operatorname{Aut}\left(L_{k}\right) \rightarrow \operatorname{Aut}\left(L_{k} / Z\right) \rightarrow 1
$$

Our main goal is to construct the lift $\xi_{k}: \operatorname{Aut}\left(L_{k} / Z\right) \rightarrow \operatorname{Aut}\left(L_{k}\right)$ (so the sequence is exact). The group $\operatorname{Tv}\left(L_{k}\right)$ can often be computed directly from a presentation of $L_{k}$, and (by Theorem 1 , say) $\operatorname{Aut}\left(L_{k} / Z\right)$ is isomorphic to the subgroup $\bar{G}_{k+1}$ of $\operatorname{Mod}\left(S_{n+2}\right)$ consisting of elements which preserve a set of $k+1$ punctures (the group $\bar{G}_{k+1}$ is generated by Dehn twists, half twists, and a reflection). In the cases of $L_{1} \cong A\left(B_{n}\right)$ and $L_{n+1} \cong P\left(A_{n}\right)$, we will show that the above exact sequence is split.

6.1. Auxiliary groups. Intuitively, we would like to "blow up" the punctures fixed by $L_{k}$ into boundary components so that the group $\bar{G}_{k+1} \cong \operatorname{Aut}\left(L_{k} / Z\right)$ cannot distinguish between the original boundary of $D_{n+1}$ and the fixed punctures. In particular, we want $\bar{G}_{k+1}$ to be able to interchange $\partial D_{n+1}$ with the fixed punctures.

We remark ahead of time that the cases of $L_{1}$ and $L_{n+1}$ are not quite as complicated as the general case of Theorem 6, even though the results are stronger for these groups (Theorems 7 and 8). Some of the details can be skipped in these cases.

Let $\bar{S}$ be a sphere with $n+2$ boundary components. We choose a set $P$ of distinguished points in $\bar{S}$, one in each boundary component. Then, we define $\overline{\operatorname{Mod}}(\bar{S})$ to be the group of homeomorphisms of $\bar{S}$ fixing $P$ as a set, modulo isotopies which fix $P$.

We fix an embedding $\bar{S} \rightarrow D_{n+1}$ which induces an isomorphism on the level of curve complexes (send each boundary component to a circle around a puncture or 
a circle parallel to $\left.\partial D_{n+1}\right)$. We will use the same names for the curves which are equivalent under this isomorphism (and the other isomorphisms below).

We get the embedding $\iota: A\left(A_{n}\right) \rightarrow \overline{\operatorname{Mod}}(\bar{S})$ as follows. If the generators $\sigma_{i}$ correspond to half twists $H_{a_{i}}$ about the 2-curves $a_{i}$, then we define $\iota\left(H_{a_{i}}\right)$ to be the generalized half twist about $a_{i}$, as indicated in Figure 5. The generalized half twist about a curve $a$ is denoted $\tilde{H}_{a}$. The homomorphism $\iota$ has appeared previously; see for instance [11].

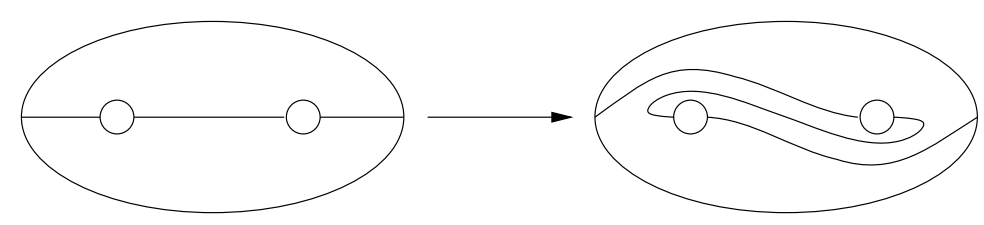

Figure 5. Generalized half twist.

For our definition of $\iota$ to be precise, we must specify the points of $P$. If $\left\{d_{i}\right\}$ are the boundary components of $\bar{S}$, we choose the unique such labelling consistent with the isomorphism $\mathcal{C}\left(D_{n+1}\right) \cong \mathcal{C}(\bar{S})$ and the choice of the $\left\{a_{i}\right\}$. We draw $\bar{S}$ in the plane so that $d_{n+2}$ is the outer boundary component and the other $d_{i}$ are Euclidean circles whose centers lie on a horizontal line. Then, the points of $P$ are chosen to be the leftmost point of each circle (this choice is consistent with Figure 5).

To see that $\iota$ is a homomorphism, one only needs to check the two braid relations. The commuting relation obviously holds. In Figure 6, we show the effect of $\iota\left(H_{a_{i}} H_{a_{i+1}} H_{a_{i}}\right)=\iota\left(H_{a_{i+1}} H_{a_{i}} H_{a_{i+1}}\right)$. We can also see that $\iota$ is injective; indeed, the map $\bar{S} \rightarrow D_{n+1}$ induces a left inverse $\pi: \overline{\operatorname{Mod}}(\bar{S}) \rightarrow A\left(A_{n}\right)$. Of course, $\iota$ restricts to an injection $L_{k} \rightarrow \overline{\operatorname{Mod}}(\bar{S})$, also called $\iota$, for any $k$.

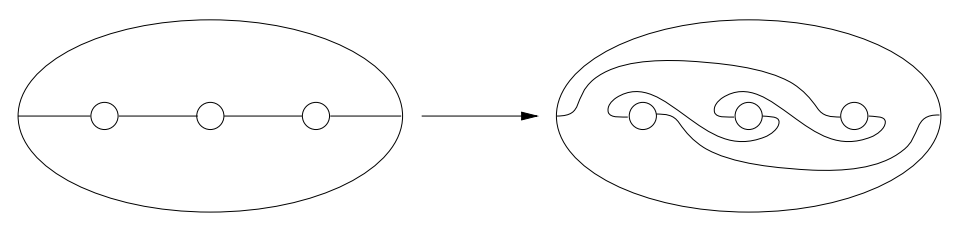

Figure 6. The braid relation in $\overline{\operatorname{Mod}}(\bar{S})$.

We introduce another surface $\bar{S}_{k}$, obtained by gluing punctured disks to the $d_{i}$ corresponding to the punctures in $D_{n+1}$ not fixed by $L_{k}$ (the surface $\bar{S}_{k}$ is a sphere with $k+1$ boundary components and $n-k+1$ punctures). The inclusion $\bar{S} \rightarrow \bar{S}_{k}$ identifies $\mathcal{C}(\bar{S})$ with $\mathcal{C}\left(\bar{S}_{k}\right)$ and induces a map $\eta: \iota\left(L_{k}\right) \rightarrow \overline{\operatorname{PMod}}\left(\bar{S}_{k}\right)$, where by $\overline{\operatorname{PMod}}\left(\bar{S}_{k}\right)$ we mean the isotopy classes of homeomorphisms of $\bar{S}_{k}$ which are the 
identity on the boundary. Fixing a set of points $P \subset \partial \bar{S}_{k}$ (one for each of the $k+1$ boundary components), we can alternatively think of $\overline{\operatorname{PMod}}\left(\bar{S}_{k}\right)$ as a normal subgroup of the group $\overline{\operatorname{Mod}}\left(\bar{S}_{k}\right)$, which consists of homeomorphisms of $\bar{S}_{k}$ fixing $P$ as a set (modulo isotopies fixing $P$ ).

The map $\iota_{k}=\left.\eta \circ \iota\right|_{L_{k}}$ is again injective since there is an inverse $\pi_{k}$ induced by gluing punctured disks to $k$ of the components of $\partial \bar{S}_{k}$.

We also want a map from $\overline{\operatorname{Mod}}\left(\bar{S}_{k}\right)$ to $\bar{G}_{k+1}$. We glue punctured disks to the $k+1$ boundary components of $\bar{S}_{k}$ in order to obtain the surface $S_{n+2}$. The inclusion of surfaces induces a surjective map $\overline{\operatorname{Mod}}\left(\bar{S}_{k}\right) \rightarrow \bar{G}_{k+1}$.

We encode the key relationships between all of our groups in Figure 7.

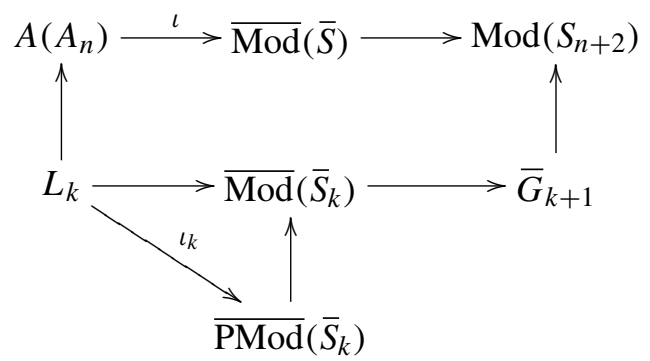

Figure 7. Groups used in the definition of $\xi_{k}: \operatorname{Aut}\left(L_{k} / Z\right) \rightarrow \operatorname{Aut}\left(L_{k}\right)$.

6.2. Generalized lantern relation. In order to define our lift $\xi_{k}: \operatorname{Aut}\left(L_{k} / Z\right) \rightarrow$ $\operatorname{Aut}\left(L_{k}\right)$, we will need a relation in $\overline{\operatorname{PMod}}(\bar{S})$ called the generalized lantern relation. Let $\left\{T_{a_{i, j}}\right\}$ be the set of Artin generators for $P\left(A_{n}\right)$, and let $\left\{d_{i}\right\}$ be the set of boundary components of $\bar{S}$ (indexed appropriately). In the language we have developed, the relation is

$$
\begin{aligned}
\iota(z) & =\iota\left(\left(T_{a_{n, n+1}}\right)\left(T_{a_{n-1, n+1}} T_{a_{n-1, n}}\right) \ldots\left(T_{a_{1, n+1}} \ldots T_{a_{1,3}} T_{a_{1,2}}\right)\right) \\
& =T_{d_{1}}^{-1} T_{d_{2}}^{-1} \ldots T_{d_{n+1}}^{-1} T_{d_{n+2}}
\end{aligned}
$$

(the first equality is the well-known relation in $P\left(A_{n}\right)$, and the second equality is the generalized lantern relation). This relation appears in the work of Wajnryb [35], who writes that the relation can be checked "by induction (by drawing many pictures)". In Section 7, we give a straightforward proof of the relation.

Without reference to $\iota$, the generalized lantern relation is simply:

$$
\left(T_{a_{n, n+1}}\right)\left(T_{a_{n-1, n+1}} T_{a_{n-1, n}}\right) \ldots\left(T_{a_{1, n+1}} \ldots T_{a_{1,3}} T_{a_{1,2}}\right)=T_{d_{1}}^{n-1} T_{d_{2}}^{n-1} \ldots T_{d_{n+1}}^{n-1} T_{d_{n+2}} .
$$

In the case of $n=2$, this relation is precisely the famous lantern relation, known to Dehn [13]. 
Stated in this alternate way, the relation exhibits an obvious asymmetry in $P\left(A_{n}\right)$ between the punctures of $D_{n+1}$ and $\partial D_{n+1}$. In our first description of the relation (which is the formulation used below), there still is an asymmetry (in the signs), and we will see that this is what prevents us from finding a homomorphism $\operatorname{Aut}\left(L_{k} / Z\right) \rightarrow$ $\operatorname{Aut}\left(L_{k}\right)$.

6.3. The lift. We now define our lift $\xi_{k}$ from $\bar{G}_{k+1} \cong \operatorname{Aut}\left(L_{k} / Z\right)$ to $\operatorname{Aut}\left(L_{k}\right)$. Given an element $f \in \bar{G}_{k+1}$, we choose a lift $\bar{f}$ in $\overline{\operatorname{Mod}}\left(\bar{S}_{k}\right)$. Since $\overline{\operatorname{PMod}}\left(\bar{S}_{k}\right)$ is normal in $\overline{\operatorname{Mod}}\left(\bar{S}_{k}\right)$, conjugation by $\bar{f}$ induces an automorphism $\psi_{f}$ of $\overline{\operatorname{PMod}}\left(\bar{S}_{k}\right)$; this automorphism is well-defined since any two lifts differ by a central element of $\overline{\operatorname{PMod}}\left(\bar{S}_{k}\right)$. We can now define an endomorphism of $L_{k}$ via the composition $\pi_{k} \circ \psi_{f} \circ \iota_{k}$. To see that this composition of homomorphisms is actually an automorphism of $L_{k}$, we will show that it is surjective. This suffices since $L_{k}$ is Hopfian, that is, every surjective endomorphism is an automorphism: braid groups are residually finite by a result of Grossman and finitely generated residually finite groups are Hopfian by results of Mal'cev [16].

The homomorphism $\pi_{k} \circ \psi_{f} \circ \iota_{k}$ clearly induces a surjection from $L_{k}$ to $L_{k} / Z$, and by the generalized lantern relation, it also induces a surjection $Z \rightarrow Z$ ( $z$ maps to either $z$ or $\left.z^{-1}\right)$. It follows that $\pi_{k} \circ \psi_{f} \circ \iota_{k}$ is a surjection. We are now justified in calling the composition $\xi_{k}(f)$, and this defines our lifting (it is clear that $\xi_{k}$ is a lift). This completes the proof of Theorem 6 .

6.4. Generalized Artin generators. The automorphisms of $L_{k}$ in the image of $\xi_{k}$ do not preserve the conjugacy classes (in $A\left(A_{n}\right)$ ) of the usual generators for $L_{k}$ : twists about 2-curves can get mapped to twists about $n$-curves. Thus, if we want to understand the image of $\xi_{k}$ via the action on generators of $L_{k}$, we need to expand the generating set.

A generalized Artin generator for $L_{k}$ is any one of the following elements of $L_{k}$ :

(1) $H_{a}$, where $a$ is a 2-curve;

(2) $T_{a}$, where $a$ is a 2-curve;

(3) $T_{a} z^{-1}$, where $a$ is an $n$-curve with a movable puncture in the exterior;

(4) $T_{a} z^{ \pm 1}$, where $a$ is an $n$-curve with a fixed puncture in the exterior.

For each of the first three types of generalized Artin generators, we denote the element associated to the curve $a$ by $g(a)$.

Below, we will argue that only the first three types are needed for $L_{1}$. For $L_{k}$ with $k>1$, the fourth type of generator is where we see the failure of $\xi_{k}$ to be a homomorphism.

Since $\iota$ is used in the definition of $\xi_{k}$, we will need to know the images under $\iota$ of the generalized Artin generators (by definition, we only know the images of the 
standard ones). Here we only deal with the first three types, since those are the ones needed for $\xi_{1}$.

By definition $\iota\left(H_{a_{1}}\right)$ is equal to $\tilde{H}_{a_{1}}$. Since $T_{a_{1,2}}=H_{a_{1}}^{2}$, one can check that $\iota\left(T_{a_{1,2}}\right)=\tilde{H}_{a_{1}}^{2}$ is equal to $T_{a_{1,2}} T_{d_{1}}^{-1} T_{d_{2}}^{-1}$ by consulting Figure 5. If $h \in A\left(A_{n}\right)$ and $h_{\star}\left(a_{1,2}\right)=a$, then we see that

$$
\iota(g(a))=\iota\left(h H_{a_{1,2}}^{q} h^{-1}\right)=\iota(h) \iota\left(H_{a_{1,2}}\right)^{q} \iota(h)^{-1}=\tilde{H}_{h_{\star}\left(a_{1,2}\right)}^{q}=\tilde{H}_{a}^{q}
$$

where $q \in\{1,2\}$. The third equality holds because $\iota(h)$ and $h$ induce the same maps of $\mathcal{C}\left(D_{n+1}\right)$ (which is identified with $\mathcal{C}(\bar{S})$ ). As part of our proof of the generalized lantern relation in Section 7, we will use our understanding of the action of $\iota$ on each $H_{a}$ and $H_{a}^{2}$ to show that if $a$ is a curve surrounding each puncture but the $i^{\text {th }}$ and $\alpha= \pm 1$, then $\iota\left(T_{a} z^{-1}\right)$ is equal to $T_{a} T_{d_{i}} T_{d_{n+2}}^{-1}$.

6.5. Automorphisms of $\boldsymbol{A}\left(\boldsymbol{B}_{n}\right)$. Recall that $A\left(B_{n}\right)$ is isomorphic to $L_{1}$, and that $A\left(B_{n}\right)$ is generated by elements $s_{i}$ where $s_{1}=T_{a_{1}}$ and $s_{i}=H_{a_{i}}$ for $i>1$. We now compute the transvection subgroup of $\operatorname{Aut}\left(A\left(B_{n}\right)\right)$ and show that our lifting $\xi_{1}$ from $\bar{G}_{2} \cong \operatorname{Aut}\left(A\left(B_{n}\right) / Z\right)$ to $\operatorname{Aut}\left(L_{1}\right) \cong \operatorname{Aut}\left(A\left(B_{n}\right)\right)$ is a homomorphism.

In Section 5, we classified all transvections of $A\left(B_{n}\right)$ in terms of two integers, $u$ and $v$. These were defined by $s_{1} \mapsto s_{1} z^{u}$ and $s_{2} \mapsto s_{2} z^{v}$. We also found that $z \mapsto z^{1+n u+n(n-1) v}$. Again, in order for a transvection to be an automorphism, we need $z \mapsto z^{ \pm 1}$. We see that $z \mapsto z$ if and only if $n u+n(n-1) v=0$ and $z \mapsto z^{-1}$ if and only if $n u+n(n-1) v=-2$. The latter case actually cannot happen, since $n u+n(n-1) v$ is divisible by $n \geq 3$ while -2 is not. Thus, $\operatorname{Tv}\left(A\left(B_{n}\right)\right) \cong \mathbb{Z}$.

We now want to show that $\xi_{1}$ is a splitting of the sequence (1). Recall that $\varepsilon: \bar{G}_{2} \rightarrow \mathbb{Z}_{2}$ is the homomorphism which records whether or not elements are orientation preserving.

We take as a generating set for $L_{1}$ the generalized Artin generators of the first three types (this contains the usual generating set). Let $f \in \bar{G}_{2} \cong \operatorname{Aut}\left(L_{1} / Z\right)$. We claim that we have the following simple formula for the action of $\xi_{1}(f)$ on the first three types of generalized Artin generators of $L_{1}$ :

$$
\xi_{1}(f)(g(a))=g\left(f_{\star}(a)\right)^{\varepsilon(f)} .
$$

It follows that $\xi_{1}$ is a homomorphism.

By the moving punctures criterion, $f$ can only switch the puncture corresponding to $\partial D_{n+1}$ with the puncture corresponding to the fixed puncture of $D_{n+1}$. Therefore, $\xi(f)$ preserves the set of generalized Artin generators of the first type. Also, $\xi(f)$ either preserves or interchanges the second and third types, depending on whether or not $f$ preserves the puncture of $S_{n+2}$ corresponding to $\partial D_{n+1}$.

Checking the above formula is straightforward. Applying $\iota_{1}$ to $g(a)$ yields a "push map" (see Section 7); composing with $\psi_{f}$ gives a push map which is conjugate in 
$\overline{\operatorname{PMod}}\left(\bar{S}_{k}\right)$; finally, $\pi_{1}$ gives $g\left(f_{\star}(a)\right)$. A more formal argument is to simply apply our computation of $\iota(g(a))$ given above. For example, if $g(a)$ is of the first type, then we have:

$$
\begin{gathered}
\iota_{1}(g(a))=\tilde{H}_{a}, \\
\psi_{f} \circ \iota_{1}(g(a))=\tilde{H}_{f_{\star}(a)}^{\varepsilon(f)}, \\
\xi_{1}(f)(g(a))=\pi_{1} \circ \psi_{f} \circ \iota_{1}(g(a))=H_{f_{\star}(f)}^{\varepsilon(a)}=g\left(f_{\star}(a)\right)^{\varepsilon(f)} .
\end{gathered}
$$

The key point is that, since $f \in \bar{G}_{2}$, and $a$ does not have the first (fixed) puncture in its interior, $f_{\star}(a)$ is also a 2-curve which does not have the first puncture in its interior. The other cases are nearly identical.

We now have that $\operatorname{Aut}\left(A\left(B_{n}\right)\right) \cong \bar{G}_{2} \ltimes \mathbb{Z}$. The group $\bar{G}_{2}$ is isomorphic to $\left(\mathbb{Z}_{2} \times \mathbb{Z}_{2}\right) \ltimes G_{2}$, where, as in the introduction, the group $G_{2}$ is the group of orientation preserving elements of $\bar{G}_{2}$ which fix two particular punctures. Thus, we can write $\operatorname{Aut}\left(A\left(B_{n}\right)\right)$ as $\left(\left(\mathbb{Z}_{2} \times \mathbb{Z}_{2}\right) \ltimes G_{2}\right) \ltimes Z$. As noted by Charney-Crisp, the elements of $G_{2}$ commute with the transvections of $\operatorname{Aut}\left(A\left(B_{n}\right)\right)$, and so, finally, we obtain Theorem 7: $\operatorname{Aut}\left(A\left(B_{n}\right)\right) \cong\left(\mathbb{Z}_{2} \times \mathbb{Z}_{2}\right) \ltimes\left(G_{2} \times \mathbb{Z}\right)$.

Remarks. In the special case of $k=1$, we can give a more straightforward definition of the lift $\xi_{k}$. Given an $f \in \bar{G}_{2}$, we define $\xi_{1}(f)$ directly by the formula $\xi_{1}(f)(g(a))=$ $g\left(f_{\star}(a)\right)^{\varepsilon(f)}$. Using the presentation of $A\left(B_{n}\right)$, and the generalized lantern relation, one can directly check that this defines a homomorphism $\bar{G}_{2} \rightarrow \operatorname{Aut}\left(A\left(B_{n}\right)\right)$.

Although not necessary for the proof, we can write down a formula for the action of $\xi_{1}(f)$ on the fourth type of generator: $\xi_{1}(f)\left(T_{a} z^{\alpha}\right)=\left(T_{f_{\star}(a)} T_{d_{n+2}}^{\alpha \delta(f)}\right)^{\varepsilon(f)}$, where $\delta$ is the homomorphism to $\mathbb{Z}_{2}$ which records the action of $f$ on the two punctures of $S_{n+2}$ which are fixed setwise by $\bar{G}_{2}$.

6.6. The case $\boldsymbol{k}>\mathbf{1}$. We now argue that $\xi_{k}$ is not a homomorphism for $k>1$. Surprisingly, there is a different lifting for the case $L_{n+1} \cong P\left(A_{n}\right)$ which is a splitting (see below).

Let $a$ be a curve in $D_{n+1}$ which surrounds all punctures except the first. Let $g \in \operatorname{Mod}\left(S_{n+2}\right) \cong \operatorname{Aut}\left(P\left(A_{n}\right) / Z\right)$ be an element whose lift $\bar{g} \in \overline{\operatorname{Mod}}(\bar{S})$ satisfies $\bar{g}\left(d_{1}\right)=d_{1}$ and $\bar{g}\left(d_{n+2}\right)=d_{2}$. Similarly to our calculations for $A\left(B_{n}\right)$, we can check that $\xi_{k}(g)$ takes $T_{a} z^{-1}$ to $T_{g_{\star}(a)}$. Let $f \in \bar{G}_{k} \cong \operatorname{Aut}\left(L_{k}\right)$ be such that $f \circ g(a)=a$, but $\bar{f} \circ \bar{g}\left(d_{1}\right)=d_{n+2}$ and $\bar{f} \circ \bar{g}\left(d_{n+2}\right)=d_{1}$. Then $\xi_{k}(f)$ takes $T_{g_{\star}(a)}$ to $T_{a} z^{-1}$. However, $\xi_{k}(f g)$ takes $T_{a} z^{-1}$ to $T_{a} z$. Thus, $\xi_{k}$ is not a homomorphism.

6.7. Automorphisms of $\boldsymbol{P}\left(\boldsymbol{A}_{\boldsymbol{n}}\right)$. A surprising and fundamental fact about $P\left(A_{n}\right)$ is that this group splits as a direct product over its center. The isomorphism $P\left(A_{n}\right) \rightarrow$ 
$P\left(A_{n}\right) / Z \times \mathbb{Z}$ is noncanonical and is given as follows.

$$
\begin{aligned}
T_{a_{1,2}} & \mapsto\left(T_{a_{1,2}}, 1\right) \\
T_{a_{i, j}} & \mapsto\left(T_{a_{i, j}}, 0\right) \quad \text { for }(i, j) \neq(1,2) .
\end{aligned}
$$

It is straightforward to check that this is an isomorphism; the key facts are that the relations for $P\left(A_{n}\right)$ are commutators and that $T_{a_{1,2}}$ appears exactly once in the standard word representing the generator for the center of $P\left(A_{n}\right)$.

In general, when a group $G$ splits over its center, its automorphism group is a semidirect product $\operatorname{Aut}(G / Z) \ltimes \operatorname{Tv}(G)$. It is a theorem of Korkmaz that $\operatorname{Aut}\left(P\left(A_{n}\right) / Z\right) \cong \operatorname{Mod}\left(S_{n+2}\right)$ (our Theorem 1 is a generalization).

The transvection subgroup can be computed directly. Using the notation of Section 5, we can choose integers $t_{i, j}$ so that $z$ maps to either $z$ or $z^{-1}$. Thus, there is a surjective map $\operatorname{Tv}\left(P\left(A_{n}\right)\right) \rightarrow \mathbb{Z}_{2}$ given by this action on the center. The kernel of this map consists of those transvections with $\sum t_{i, j}=0$. As in Section 5, there are $N=\left(\begin{array}{c}n+1 \\ 2\end{array}\right)$ of the $t_{i, j}$, and so this kernel is isomorphic to $\mathbb{Z}^{N-1}$. There is a splitting $\mathbb{Z}_{2} \rightarrow \operatorname{Tv}\left(P\left(A_{n}\right)\right.$ ) (set $t_{1,2}=-2$ and all other $t_{i, j}=0$ ), and so $\operatorname{Tv}\left(P\left(A_{n}\right)\right) \cong \mathbb{Z}_{2} \ltimes \mathbb{Z}^{N-1}$. This completes the proof of Theorem 8 .

\section{Generalized lantern relation}

We now prove the "generalized lantern relation", used in Section 6. We freely use the notation of that section. Also, we make a point of recalling that, contrary to the usual convention for braid groups, we use functional notation for the composition of mapping classes.

Our goal is to understand $\iota(z)$. We think of $z$ as a product of elements

$$
g_{i}=T_{a_{i, n+1}} \ldots T_{a_{i, i+2}} T_{a_{i, i+1}}
$$

and we will first understand each $\iota\left(g_{i}\right)$ individually. We draw $\bar{S}$ in the plane as in Section 6. This allows us to see the $a_{i, j}$ in $\bar{S}$ exactly as they appear in $D_{n+1}$.

We can think of each $T_{a_{i, j}}$ as a push map, where the $i^{\text {th }}$ boundary component moves around the $j^{\text {th }}$ boundary component, while travelling clockwise inside $a_{i, j}$ in such a way that it never turns (Figure 5 represents the halfway point).

We can thus think of $g_{i}$ as a product of these push maps (the solid arrows in Figure 8). We now see the following intuitive relation: $g_{i}$ can also be obtained by pushing the $i^{\text {th }}$ boundary component around the $n-i+1$ boundary components to its right all at once (the dashed arrow in Figure 8). We then observe that this latter push map is equivalent to

$$
T_{c_{i+1, n+1}}^{-1} T_{d_{i}}^{-1} T_{c_{i, n+1}}
$$


$\circ$

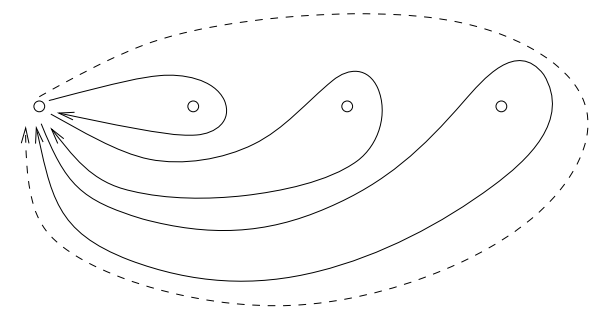

Figure 8. A relation between push maps.

where the curves are as shown in Figure 9. This intuitive relation (which is already an interesting relation in the mapping class group) is explained more formally in the remark below (see also [30], [33]).

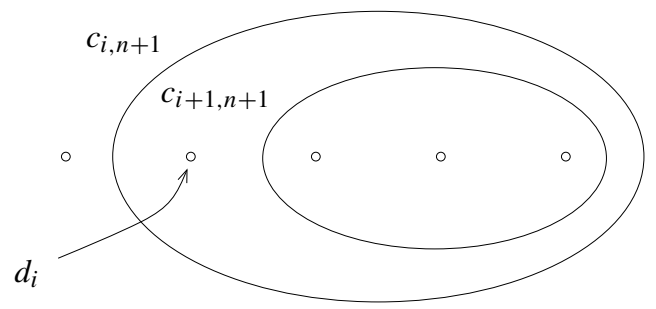

Figure 9. The curves needed to write $g_{i}$ as a product of Dehn twists.

We can now compute $\iota(z)$ as the product of the $\iota\left(g_{i}\right)$ :

$$
\left(T_{c_{n+1, n+1}}^{-1} T_{d_{n}}^{-1} T_{c_{n, n+1}}\right) \ldots\left(T_{c_{3, n+1}}^{-1} T_{d_{2}}^{-1} T_{c_{2, n+1}}\right)\left(T_{c_{2, n+1}}^{-1} T_{d_{1}}^{-1} T_{c_{1, n+1}}\right) .
$$

All of the $T_{c_{i, j}}$ elements cancel except the first (rightmost), which is equal to $T_{d_{n+2}}$, and the last, which is equal to $T_{d_{n+1}}^{-1}$. Thus, $\iota(z)$ is equal to the product of $T_{d_{n+2}}$ with $T_{d_{1}}^{-1} \ldots T_{d_{n+1}}^{-1}$, and this is exactly the generalized lantern relation.

We notice that, applying the map $\pi$ to $\iota(z)$, we see that we have proven that the product of the $g_{i}$ is indeed equal to $z$ in $P\left(A_{n}\right)$.

Also, since $\iota$ takes conjugates of the $T_{a_{i, j}}$ to the corresponding conjugates of the $\iota\left(T_{a_{i, j}}\right)$, the same holds for the conjugates of the $g_{i}$. This fact was used in Section 6.

Remark. We now explain a more formal framework for proving the intuitive relation that pushing a disk around two loops is the same as pushing it around a composite loop. Let $X$ be the subset of the configuration space of $n+1$ ordered points in the unit tangent bundle of the disk, where each point lies in a different fiber. There is a natural map from $\pi_{1}(X) \rightarrow \overline{\operatorname{Mod}}(\bar{S})$ (the projection to the disk of each point in $X$ specifies the location of a particular boundary component and the vector specifies 
the rotation; if we like, we can replace boundary components with rigid disks in the sphere). The relation described above simply follows from the fact that this map is a homomorphism. Putman has observed that relations in $\pi_{1}(X)$ thus give rise to many different "generalized lantern relations" [33].

\section{Appendix. Superinjective maps of curve complexes}

In this section, let $S=S_{g, n}$ be an orientable surface of genus $g$ with $n$ punctures. Throughout, we tacitly assume that all surfaces have negative Euler characteristic (the curve complex is uninteresting otherwise). We define the complexity of $S_{g, n}$ to be $3 g+n-3$; for the surfaces under consideration this is the number of curves in a pants decomposition.

We define the curve complex $\mathcal{C}(S)$ as the abstract simplicial flag complex with vertices corresponding to isotopy classes of essential curves in $S$ (or simply "curves") and edges corresponding to curves in $S$ which have the smallest possible geometric intersection number in $S$. For $S_{0,4}$, the minimal intersection is 2 ; for $S_{1,1}$, the minimal intersection is 1; and for all other $S$ with $\mathcal{C}(S)$ nonempty, the minimal intersection is zero, as in the main body of this paper.

A.1. Statement of theorem. Our main goal is to prove the following.

Theorem 16. Let $S=S_{g, n}$ and $S^{\prime}=S_{g^{\prime}, n^{\prime}}$ be two surfaces of the same complexity. Any superinjective map $\phi: \mathcal{C}(S) \rightarrow \mathcal{C}\left(S^{\prime}\right)$ is surjective.

Superinjective maps are easily seen to be injective (given two vertices, there is a vertex connected to one but not the other), so it follows that superinjective maps are automorphisms. We can then apply the theorem of Ivanov, Korkmaz, and Luo that if $S$ is any surface of negative Euler characteristic other than $S_{1,2}$, then each automorphism of $\mathcal{C}(S)$ is induced by $\operatorname{Mod}(S)$ [22], [26], [28]. The result is that, for these surfaces, superinjective maps of $\mathcal{C}(S)$ are induced by $\operatorname{Mod}(S)$; this is the general case of Theorem 2. As a consequence, one can deduce the corresponding generalization of Theorem 1 (see [3] for precise statements).

Theorem 16 actually implies a more general theorem, due to Shackleton: there are no injective homomorphisms from a finite index subgroup of the mapping class group of one surface to a mapping class group of another surface with equal or smaller complexity, if the curve complexes are not isomorphic [34].

We also give an elementary proof of the classification of curve complexes [28]; that is, the only isomorphic curve complexes are

$$
\mathcal{C}\left(S_{1,1}\right) \cong \mathcal{C}\left(S_{0,4}\right), \quad \mathcal{C}\left(S_{1,2}\right) \cong \mathcal{C}\left(S_{0,5}\right), \quad \mathcal{C}\left(S_{2,0}\right) \cong \mathcal{C}\left(S_{0,6}\right) .
$$


Historical remark. There are a number of papers on automorphisms and superinjective maps of the curve complex. Different versions of this paper appeared at various points in the progression of ideas. The theorems stated in the introduction were new theorems when the first version of this paper was written. The more general statements and proofs given in this appendix came later. We keep the old statements in the introduction to preserve this paper's place in the history, but present the newer proofs in this appendix because they are both simpler and more general.

In brief, Ivanov first proved the automorphism theorem for surfaces of genus at least 2 [22], then Korkmaz proved it for genus 0 and 1 [26]. Around the same time, Luo gave a completely different proof for all surfaces, and also settled the case of the twice punctured torus [28]. Irmak proved the superinjective theorem for surfaces of genus at least 2 [18], [20], [19]. Later, the first version of this paper proved the superinjective theorem for genus 0 [5], and Behrstock-Margalit proved it for genus 1 [3]. Finally, Shackleton proved that an injective simplicial map between curve complexes of the same dimension is an isomorphism [34].

A.2. Proof of theorem. We now begin our proof of Theorem 16, which is an induction on complexity. First we deal with the lowest complexity cases. For the surface of complexity 0 , namely $S_{0,3}$, Theorem 16 is vacuously true, since $\mathcal{C}\left(S_{0,3}\right)$ is empty.

Farey graphs. For surfaces of complexity $1\left(S_{1,1}\right.$ and $\left.S_{0,4}\right)$, Theorem 16 is a well known fact. In these cases, $\mathcal{C}(S)$ is the Farey graph, or ideal triangulation of the disk (see [32]), and one readily checks that any injective simplicial map of the Farey graph is surjective.

Lemma 17. Let $S$ and $S^{\prime}$ be complexity 1 surfaces. Every superinjective map $\phi: \mathcal{C}(S) \rightarrow \mathcal{C}\left(S^{\prime}\right)$ is surjective.

From this point forward, let $S$ and $S^{\prime}$ be fixed surfaces of the same complexity $\geq 2$, and let $\phi: \mathcal{C}(S) \rightarrow \mathcal{C}\left(S^{\prime}\right)$ be a fixed superinjective map.

Adjacency graph. To proceed by induction on complexity, the main step is to show that $\phi$ preserves the complexity of a curve $c$, by which we mean the minimum positive complexity of a component of $S-c$. A useful tool for this is the adjacency graph, which is a graph $\mathscr{g}(\mathcal{P})$ associated to a pants decomposition $\mathcal{P}$. It has a vertex for every element of $\mathcal{P}$, and an edge if the corresponding curves are boundary components of the same pair of pants in $S$. Note that $g(\mathcal{P})$ is always connected.

Since $\phi$ is injective, it maps a pants decomposition $\mathcal{P}$ to a pants decomposition $\phi(\mathcal{P})$ and induces a map from the vertices of $g(\mathcal{P})$ to the vertices of $g(\phi(\mathcal{P}))$. In fact, more is true.

Lemma 18. Let $\mathcal{P}$ be a pants decomposition for $S$. The map $\phi$ induces a graph isomorphism $g(\mathcal{P}) \rightarrow g(\phi(\mathcal{P}))$. 
The adjacency graph was introduced by Behrstock-Margalit, and also by Shackleton [3], [34].

Proof. Let $a$ and $b$ be two curves and $\mathcal{P}=\left\{a, b, c_{1}, \ldots, c_{k}\right\}$ a pants decomposition for $S$. If $a$ and $b$, say, are connected by an edge in $g(\mathcal{P})$, then we can find a vertex $z$ of $\mathcal{C}(S)$ which is not connected to $a$ or $b$ but is connected to $c_{i}$ for all $i$, i.e., $z$ intersects $a$ and $b$ but no other elements of $\mathcal{P}$ (we identify vertices of $\mathcal{g}(\mathcal{P})$ with vertices of $\mathcal{C}(S)$ and with curves in $S)$. Thus, $\phi(a)$ and $\phi(b)$ must be connected by an edge in $\mathcal{G}(\phi(\mathcal{P}))$.

If $a$ and $b$ are not connected by an edge in $g_{(}(\mathcal{P})$, then there are curves $a_{1}$ and $b_{1}$ so that, if we set $a_{0}=a$ and $b_{0}=b$, the set of curves $\left\{a_{i}, b_{j}, c_{1}, \ldots, c_{k}\right\}$ is a pants decomposition for any of the 4 choices for $i$ and $j$. In other words, $\left(a_{0}, b_{0}, a_{1}, b_{1}\right)$, in that order, form a square in $\mathcal{C}\left(S-\cup c_{i}\right)$. If $\phi(a)$ and $\phi(b)$ are connected by an edge in $g(\phi(\mathcal{P})$ ), then $\phi(a)$ and $\phi(b)$ lie on a connected complexity 2 component of $S^{\prime}-\cup \phi\left(c_{i}\right)$. However, $\mathcal{C}\left(S_{0,5}\right) \cong \mathcal{C}\left(S_{1,2}\right)$ contains no squares. Thus, $\phi(a)$ and $\phi(b)$ cannot be connected by an edge in $\mathscr{g}(\phi(\mathcal{P}))$.

As an immediate consequence, we have the desired lemma about curve complexity.

Lemma 19. For any curve $c, \phi(c)$ has the same complexity as $c$.

Proof. Let $c$ be any curve in $S$ and $\mathcal{P}$ any pants decomposition containing $c$. Complexity can be read off from $\mathscr{g}(\mathcal{P})$-remove the vertex $c$ and all (open) edges emanating from $c$, and count the number of vertices in each of the remaining components. Thus, an application of Lemma 18 completes the proof.

Simple pairs. Two curves $a$ and $b$ form a simple pair in $S$ if there is a pants decomposition $\mathcal{P}=\left\{a, c_{0}, \ldots, c_{k}\right\}$ so that $b$ is also disjoint from the $c_{i}$, and $a$ and $b$ are connected by an edge in the curve complex for the complexity 1 component of $S-\cup c_{i}$. The following lemma was proven in the case of nonseparating curves by Ivanov in his original work concerning automorphisms of $\mathcal{C}(S)$ [23, Lemma 1].

Lemma 20. If $a$ and $b$ form a simple pair of curves in $S$, then $\phi(a)$ and $\phi(b)$ form $a$ simple pair.

Proof. Let $\mathcal{P}=\left\{a, c_{0}, \ldots, c_{k}\right\}$ be a pants decomposition which comes from the definition of simple pairs. We assume that $a$ and $c_{0}$ are connected in $g(\mathcal{P})$, and we consider the complexity 2 component $F$ of $S-\left\{c_{1}, \ldots, c_{k}\right\}$. Let us first assume that $F$ is $S_{0,5}$.

We claim that $a$ and $b$ form a simple pair if and only if they are nonadjacent points of some pentagon in $\mathcal{C}\left(S_{0,5}\right)$. One direction is easily exhibited by construction. The other direction is left as an exercise; see [4] for a proof. 
Since $\phi$ preserves all of the properties used in this characterization of simple pairs, we are done in the case of $S_{0,5}$. Since $\mathcal{C}\left(S_{0,5}\right) \cong \mathcal{C}\left(S_{1,2}\right)$, and the isomorphism takes simple pairs to simple pairs (the isomorphism is induced by the hyperelliptic involution of $S_{1,2}$; see [28]), the proof is complete.

We are now ready to prove the theorem.

Proof of Theorem 16. As advertised, we proceed by induction on the complexity of $S$. The base step is Lemma 17. Now suppose that the complexity of $S$ is greater than 1, and that the theorem is true for all surfaces of smaller complexity.

Let $c$ be any curve in $S$. By Lemma 19, $\phi(c)$ has the same complexity. It then follows from consideration of the adjacency graph that $\phi$ induces maps from the curve complexes of the component(s) of $S-c$ to the curve complexes of the components of $S^{\prime}-\phi(c)$. What is more, the induced maps are superinjective: for components of complexity 1 this follows from Lemma 20 and for components of complexity greater than 1 it is obvious (we simply throw out components of complexity zero). The components of $S-c$ have complexity strictly smaller than that of $S$ and so by induction $\phi$ restricts to surjective maps of the corresponding curve complexes.

As a result, we see that for every curve in $S^{\prime}$ which is in the image of $\phi$, the closed star of that vertex in $\mathcal{C}\left(S^{\prime}\right)$ is in the image of $\phi$. Since $\mathcal{C}\left(S^{\prime}\right)$ is connected, $\phi$ is surjective.

A.3. Classification. We now apply Lemmas 19 and 20 to give a simple proof of the classification of isomorphic curve complexes. Of course, automorphisms are superinjective, so we may freely use these lemmas.

For the proof, we define a standard simple pair to be a simple pair which lies on a complexity 1 side of a complexity 1 curve. It follows from Lemmas 18, 19, and 20 that standard simple pairs are preserved by automorphisms of $\mathcal{C}(S)$. The key feature is that, except in the case of $S_{1,2}$, the curves of a standard simple pair are of the same topological type: either both nonseparating, or both curves which cut off a twice-punctured disk.

Theorem 21. Aside from the pairs $\left(S_{1,1}, S_{0,4}\right),\left(S_{1,2}, S_{0,5}\right)$, and $\left(S_{2,0}, S_{0,6}\right)$, no two surfaces of negative Euler characteristic have isomorphic curve complexes.

In each of the three exceptional cases, the isomorphism is induced by the hyperelliptic involution of the surface of positive genus (see [28]).

Proof. Since isomorphic curve complexes have the same dimension, it suffices to show that the genus of $S=S_{g, n}$ can be detected in $\mathcal{C}(S)$. Given the list of isomorphic curve complexes, we may assume that the complexity of $S$ is at least 3, and that $S$ is not $S_{2,0}$.

The first step is to show that nonseparating curves can be recognized in $\mathcal{C}(S)$. Nonseparating curves are almost characterized by the fact that they are not cut points in 
any adjacency graph; in other words, their complexity is one less than the complexity of $S$. The only other curves which satisfy this property are curves which cut off a twice-punctured disk in $S$. One difference between these two kinds of curves is that a nonseparating curve can form a standard simple pair with three disjoint curves simultaneously (this fails for $S_{2,0}$ ), while for a curve which bounds a twice-punctured disk, this is impossible.

But now the genus of $S$ is simply the maximum number of disjoint standard simple pairs of nonseparating curves, so we are done.

A.4. Automorphisms. Finally, we say a few words about the proofs of the theorem that automorphisms of $\mathcal{C}(S)$ are induced by $\operatorname{Mod}(S)$. Ivanov's original approach was to show that an automorphism of $\mathcal{C}(S)$ induces an automorphism of an associated arc complex, and then apply the fact that automorphisms of the arc complex are determined by the action on a single maximal simplex. Luo gives an inductive proof, also making use of an algebraic structure on the vertices of $\mathcal{C}(S)$ : two curves are "multiplied" via surgery, and so it suffices to understand the automorphism on a generating set.

Building on this idea, Shackleton gives a purely topological inductive approach as follows. Let $a$ be a curve in $S$. Since the topological type of $a$ is preserved (as above), there is an induced automorphism of $\mathcal{C}(S-a)$ which, by induction, is induced by $\operatorname{Mod}(S-a)$. To this element of $\operatorname{Mod}(S-a)$ corresponds a $\left\langle T_{a}\right\rangle$ coset in $\operatorname{Mod}(S)$. To pin down a particular element, one repeats the procedure with a curve $b$ disjoint from $a$. The intersection of the two cosets is a single mapping class, which, a priori, depends on $a$ and $b$. One then chooses a curve $c$ which is connected to $b$ in $\mathcal{C}(S-a)$ and shows that the mapping class associated to $a, b$ is the same as that associated to $a, c$. By connectedness of $\mathcal{C}(S)$, the proof is complete. For details, see Shackleton's paper (what he calls "the inductive step") [34, pages 229-231].

\section{References}

[1] Daniel Allcock, Braid pictures for Artin groups. Trans. Amer. Math. Soc. 354 (9) (2002), 3455-3474. Zbl 1059.20032 MR 1911508

[2] E. Artin, Theory of braids. Ann. of Math. (2) 48 (1947), 101-126. Zbl 0030.17703 MR 0019087

[3] Jason Behrstock and Dan Margalit, Curve complexes and finite index subgroups of mapping class groups. Geom. Dedicata 118 (2006), 71-85. Zbl 05046839 MR 2239449

[4] Robert W. Bell and Dan Margalit, Braid groups and the co-Hopfian property. J. Algebra 303 (1) (2006), 275-294. Zbl 1110.20028 MR 2253663

[5] Robert W. Bell and Dan Margalit, Injections of Artin groups (version 2). Preprint, 2006; arXiv:math.DG/0501051. 
[6] Joan S. Birman and Tara E. Brendle, Braids: a survey. In Handbook of knot theory, Elsevier B. V., Amsterdam 2005, 19-103. Zbl 1094.57006 MR 2179260

[7] Joan S. Birman, Alex Lubotzky, and John McCarthy, Abelian and solvable subgroups of the mapping class groups. Duke Math. J. 50 (4) (1983), 1107-1120. Zbl 0551.57004 MR 0726319

[8] Tara E. Brendle and Dan Margalit, Commensurations of the Johnson Kernel. Geom. Topol. 8 (2004), 1361-1384. Zbl 1079.57017 MR 2119299

[9] Ruth Charney and John Crisp, Automorphism groups of some affine and finite type Artin groups. Math. Res. Lett. 12 (2-3) (2005), 321-333. Zbl 1077.20055 MR 2150887

[10] Ruth Charney and David Peifer, The $K(\pi, 1)$-conjecture for the affine braid groups. Comment. Math. Helv. 78 (3) (2003), 584-600. Zbl 1066.20043 MR 1998395

[11] John Crisp and Luis Paris, Representations of the braid group by automorphisms of groups, invariants of links, and Garside groups. Pacific J. Math. 221 (1) (2005), 1-27. Zbl 05039939 MR 2194143

[12] John Crisp, Injective maps between Artin groups. In Geometric group theory down under (Canberra, 1996), Walter de Gruyter, Berlin 1999, 119-137. Zbl 1001.20034 MR 1714842

[13] Max Dehn, Papers on group theory and topology. Springer-Verlag, New York 1987. MR 0881797

[14] Joan L. Dyer and Edna K. Grossman, The automorphism groups of the braid groups. Amer. J. Math. 103 (6) (1981), 1151-1169. Zbl 0476.20026 MR 0636956

[15] Benson Farb and Nikolai V. Ivanov, The Torelli geometry and its applications: research announcement. Math. Res. Lett. 12 (2-3) (2005), 293-301. Zbl 1073.57012 MR 2150885

[16] Edna K. Grossman, On the residual finiteness of certain mapping class groups. J. London Math. Soc. (2) 9 (1974/75), 160-164. Zbl 0292.20032 MR 0405423

[17] W. J. Harvey, Boundary structure of the modular group. In Riemann surfaces and related topics: Proceedings of the 1978 Stony Brook Conference (State Univ. New York, Stony Brook, N.Y., 1978), Ann. of Math. Stud. 97, Princeton University Press, Princeton, N.J., 1981, 245-251. Zbl 0461.30036 MR 0624817

[18] Elmas Irmak, Superinjective simplicial maps of complexes of curves and injective homomorphisms of subgroups of mapping class groups. Topology 43 (3) (2004), 513-541. Zbl 1052.57024 MR 2041629

[19] Elmas Irmak, Complexes of nonseparating curves and mapping class groups. Michigan Math. J. 54 (1) (2006), 81-110. Zbl 05140512 MR 2214789

[20] Elmas Irmak, Superinjective simplicial maps of complexes of curves and injective homomorphisms of subgroups of mapping class groups. II. Topology Appl. 153 (8) (2006), 1309-1340. Zbl 1100.57020 MR 2202856

[21] Nikolai V. Ivanov and John D. McCarthy, On injective homomorphisms between Teichmüller modular groups. I. Invent. Math. 135 (2) (1999), 425-486. Zbl 0978.57014 MR 1666775

[22] Nikolai V. Ivanov, Subgroups of Teichmüller modular groups, Transl. Math. Monogr. 115, Amer. Math. Soc., Providence, RI, 1992. Zbl 0776.57001 MR 1195787

[23] Nikolai V. Ivanov, Automorphism of complexes of curves and of Teichmüller spaces. Internat. Math. Res. Notices 1997 (14) (1997), 651-666. Zbl 0890.57018 MR 1460387 
[24] N. V. Ivanov, Automorphisms of Teichmüller modular groups. In Topology and geometry—Rohlin Seminar, Lecture Notes in Math. 1346, Springer, Berlin 1988, 199-270. Zbl 0657.57004 MR 0970079

[25] Richard P. Kent, IV and David Peifer, A geometric and algebraic description of annular braid groups. Internat. J. Algebra Comput. 12 (1-2) (2002), 85-97. Zbl 1010.20024 MR 1902362

[26] Mustafa Korkmaz, Automorphisms of complexes of curves on punctured spheres and on punctured tori. Topology Appl. 95 (2) (1999), 85-111. Zbl 0926.57012 MR 1696431

[27] Christopher J. Leininger and Dan Margalit, Abstract commensurators of braid groups. J. Algebra 299 (2) (2006), 447-455. Zbl 1103.20034 MR 2228321

[28] Feng Luo, Automorphisms of the complex of curves. Topology 39 (2) (2000), 283-298. Zbl 0951.32012 MR 1722024

[29] Wilhelm Magnus, Abraham Karrass, and Donald Solitar, Combinatorial group theory. Reprint of the second edition, Dover Publications Inc., Mineola, N.Y., 2004. Zbl 02146477 MR 2109550

[30] Dan Margalit and Jon McCammond, Geometric presentations for the pure braid group. Preprint, 2006. arXiv:math.GT/0603204.

[31] John D. McCarthy and William R. Vautaw, Automorphisms of Torelli groups. Preprint, 2003. arXiv:math.GT/0311250

[32] Yair N. Minsky, A geometric approach to the complex of curves on a surface. In Topology and Teichmüller spaces (Katinkulta, 1995), World Sci. Publishing, River Edge, N.J., 1996, 149-158. Zbl 0937.30027 MR 1659683

[33] Andrew Putman, An infinite presentation of the Torelli groups. Preprint, 2007. arXiv:math.GT/0703529

[34] Kenneth J. Shackleton, Combinatorial rigidity in curve complexes and mapping class groups. Pacific J. Math. 230 (1) (2007), 217-232.

[35] Bronisław Wajnryb, Mapping class group of a handlebody. Fund. Math. 158 (3) (1998), 195-228. Zbl 0928.57013 MR 1663329

Received January 6, 2005

Robert Bell, A-305 Wells Hall, Department of Mathematics, Michigan State University, East Lansing, MI 48824, U.S.A.

E-mail: rbell@math.msu.edu

Dan Margalit, Department of Mathematics, University of Utah, 155 S 1440 East,

Salt Lake City, UT 84112-0090, U.S.A.

E-mail: margalit@math.utah.edu 